\title{
A Simple Method of H/He Influx Ratio Measurement as a Monitor for Machine Operation in LHD
}

\author{
Shigeru MORITA, Motoshi GOTO, Suguru MASUZAKI, Naoko ASHIKAWA \\ and LHD experimental group \\ National Institute for Fusion Science, Toki 509-5292, Gifu, Japan
}

(Received 20 November 2007 / Accepted 5 March 2008)

\begin{abstract}
In order to observe edge neutral recycling for fusion machine operation, the absolute values of $\mathrm{H}_{\alpha}$ and HeI emissions have been usually analyzed with measurement of edge density and temperature. On the other hand, it is known that the temperature and density dependences of each line can be negated by taking the ratio between the two lines. The intensity ratio of $\mathrm{H}_{\alpha}(6563 \AA)$ to HeI (5876 $\mathrm{A}$ or $6678 \AA$ ) visible spectral lines is adopted, and the results are presented here instead of the method usually used for monitoring the edge particle recycling and the effect of wall conditioning in LHD. The ionization events per photon were calculated for both emissions using a collisional-radiative model, and the ratio of $\mathrm{H}^{+}$flux ( $\equiv \mathrm{H}^{0}$ influx) to $\mathrm{He}^{+}$flux ( $\equiv \mathrm{He}^{0}$ influx) was obtained. The $\mathrm{H}_{\alpha}$ and HeI (5876 $\AA$ ) emissions in LHD have been measured using a monitor assembly with an interference filter and optical fiber array. The $\mathrm{H}^{+} / \mathrm{He}^{+}$flux ratio has been thus evaluated by integrating the $\mathrm{H}_{\alpha}$ and HeI emissions from a 10-channel toroidal array. As an example of the measurement, all discharges performed during the past 9 years in LHD have been analyzed. The present method shows that the aftereffects of the $\mathrm{H}_{2}$ and He glow discharge cleaning for vacuum wall conditioning on the LHD discharges become clearly visible. The replacement of deposited atoms on the carbon divertor plates was also analyzed. It is found that the replacement is completed by $40-50$ shot repetition of discharges. These results indicate the effectiveness of the present method as a good monitor for fusion machine operation.
\end{abstract}

(c) 2008 The Japan Society of Plasma Science and Nuclear Fusion Research

Keywords: $\mathrm{H}_{\alpha}, \mathrm{HeI}, \mathrm{H} / \mathrm{He}$ ratio, wall conditioning, plasma facing material, visible spectroscopy, LHD

DOI: $10.1585 /$ pfr.3.S1037

\section{Introduction}

Wall conditioning in fusion devices is one of the experimentally important techniques for improving plasma performance. The edge temperature rise based on the reduction of particle recycling is the main purpose of wall conditioning. In LHD, the wall conditioning becomes more important since the baking temperature of the vacuum vessel is limited to $95^{\circ} \mathrm{C}$ because of a narrow space between the superconducting helical coils and the vacuum vessel. In LHD, therefore, a variety of wall conditioning techniques such as $T_{i}$ gettering, low magnetic field ECR discharge, boronization, and $\mathrm{H}_{2}, \mathrm{He}$, and $\mathrm{Ne}$ glow discharges have been attempted until now, and the He glow discharge has been finally selected as the main method [1-3]. Although the He glow discharge was very effective in removing the hydrogen from the wall and divertor plates, a large amount of He unexpectedly appeared as the enhanced influx in the $\mathrm{H}_{2}$ discharge. It becomes then important to measure the aftereffects of such discharge cleanings and the shot-by-shot variation in edge recycling.

In order to monitor the hydrogen and helium influxes and their toroidal uniformity, on the other hand, toroidal distributions of $\mathrm{H}_{\alpha}$ and HeI visible emissions have been measured with a toroidal array system in LHD. When their influxes are analyzed, the edge $n_{\mathrm{e}}$ and $T_{\mathrm{e}}$ profiles are always necessary for influx calculation in addition to the absolute values of the $\mathrm{H}_{\alpha}$ and $\mathrm{HeI}$ emissions. However, it is really difficult to analyze the influxes in all discharges, because the edge parameter range distributes very widely due to the presence of an ergodic layer, and the data have to be then basically checked on a shot-by-shot basis $[4,5]$. Therefore, a new method with a simpler technique was required to monitor the status of wall conditioning.

The temperature and density dependences of spectral lines can be negated when the ratio between two appropriate lines is taken. The $\mathrm{H}_{\alpha}(6563 \AA)$ and HeI $(5876 \AA$ or $6678 \AA$ ) emissions have similar temperature and density dependences in a general edge plasma condition because they have the same transition of $3 d-2 p$. It is expected that the large dependences on temperature and density can be negated when the intensity ratio of $\mathrm{H}_{\alpha}$ to $\mathrm{HeI}$ is taken and the ratio becomes a good measure to monitor the vacuum wall status of fusion devices. The $\mathrm{H}_{\alpha} / \mathrm{HeI}$ intensity ratio was then newly used for the purpose. In order to check the effectiveness of the method, the flux ratio of $\mathrm{H}^{+}$to $\mathrm{He}^{+}$ ions evaluated from the $\mathrm{H}_{\alpha} / \mathrm{HeI}$ intensity ratio is analyzed for all discharges over 9 years in LHD, and the shot-by- 
shot behavior of the ratio is also analyzed in a few cases. In this paper, a convenient method to monitor the vacuum wall status is reported with analyses of the $\mathrm{H}^{+} / \mathrm{He}^{+}$flux ratio in LHD.

\section{Calculation of $\mathrm{H}^{+} / \mathrm{He}^{+}$Ion Flux Ratio}

The emission rates of the $\mathrm{H}_{\alpha}$ and HeI lines are generally functions of $T_{\mathrm{e}}$ and $n_{\mathrm{e}}$. The population of excited levels in neutral hydrogen and helium is then calculated using a collisional-radiative model $[6,7]$. The coefficient of ionization events per photon is needed for calculating the hydrogen and helium neutral influxes in addition to the emission intensity itself. Here, the ionization events per photon give the conversion rate from the neutral emission to the ion flux. The results for $\mathrm{H}_{\alpha}\left(2 \mathrm{p}^{2} \mathrm{P}-3 \mathrm{~d}^{2} \mathrm{D}: 6563 \AA\right)$ emission are shown in Fig. 1 as a function of electron density. The calculation is performed for four different electron temperatures as a parameter. In the LHD experiment, on the other hand, the radial profile of the $\mathrm{H}_{\alpha}$ emission has been measured. It is then confirmed that the electron temperature at the location where the $\mathrm{H}_{\alpha}$ line is emitted ranges from 10 to $100 \mathrm{eV}$, except for the recombining phase after switching off the heating devices [8]. From Fig. 1, it is understood that the temperature dependence of the ionization events per photon is really weak in the $10-100 \mathrm{eV}$ range. However, the density dependence becomes considerably large, especially at densities greater than $1 \times 10^{13} \mathrm{~cm}^{-3}$, whereas the ionization events per photon for $\mathrm{H}_{\alpha}$ emission have been frequently used as a constant $[9,10]$.

The two neutral helium transitions of $2 \mathrm{p}^{3} \mathrm{P}-3 \mathrm{~d}^{3} \mathrm{D}$ (5876 $\AA$ ) and $2 \mathrm{p}^{1} \mathrm{P}-3 \mathrm{~d}^{1} \mathrm{D}(6678 \AA)$ are calculated and the results are shown in Figs. 2 (a) and (b), respectively. The ionization events per photon of HeI $5876 \AA$ in triplet sys-

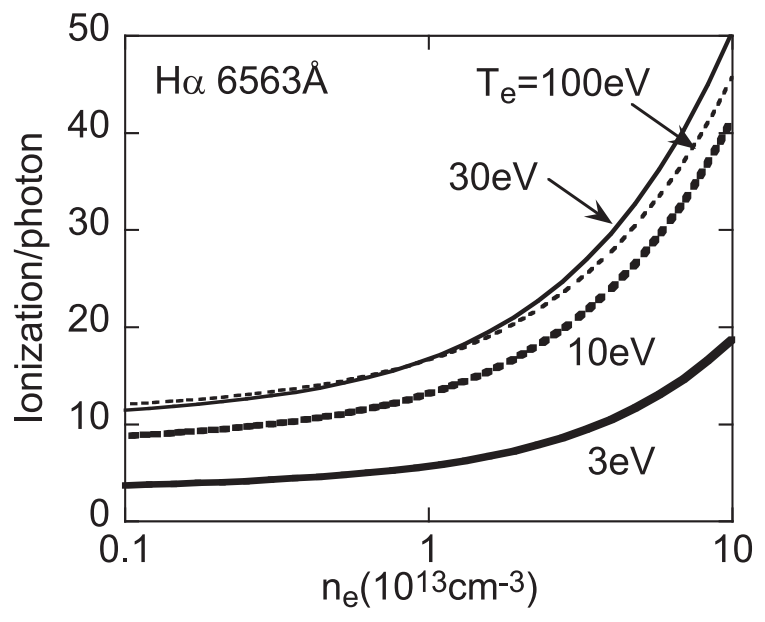

Fig. 1 Ionization events per photon for $2 \mathrm{p}^{2} \mathrm{P}-3 \mathrm{~d}^{2} \mathrm{D} \mathrm{H}_{\alpha}$ emission as a function of electron density. Calculation is performed for four different electron temperatures (thick solid line: $3 \mathrm{eV}$, thick dashed line: $10 \mathrm{eV}$, thin solid line: $30 \mathrm{eV}$, and thin dashed line: $100 \mathrm{eV}$ ). tem have stronger density and temperature dependences than those of HeI $6678 \AA$ in a singlet system. In particular, this tends to be emphasized when exceeding the density of $1 \times 10^{13} \mathrm{~cm}^{-3}$. This strong density dependence in the triplet system originates in a weak radiative decay rate from the $n=2$ level, and as a result, a metastable state appeared at the $n=2$ level.

The ratio of hydrogen ion flux to total ion flux (三 sum of hydrogen and helium ion fluxes) is shown in Figs. 3 (a) and (b) for HeI $5876 \AA$ and HeI $6678 \AA$, respectively. The flux from impurity ions is ignored in the present analysis. Here, the $\mathrm{H}^{+}$(or $\mathrm{He}^{+}$) flux means the physical value identical to the $\mathrm{H}$ (or $\mathrm{He}$ ) neutral influx, which is usually calculated from the ionization events per photon. In this paper, the $\mathrm{H}^{+}$(or $\mathrm{He}^{+}$) flux is used instead of the neutral influx to have a more realistic image in edge particle recycling. It is also noticed that the neutral influx does not exactly mean the direct influx to the main plasma. This should be further emphasized in the LHD magnetic geometry, since the main plasma is surrounded by a thick ergodic layer. The calculation is performed for $\mathrm{H}_{\alpha}$ and HeI emissions having the same photon number. It is clear that the edge parameter dependence considerably disappears when the ratio of $\mathrm{H}_{\alpha}$

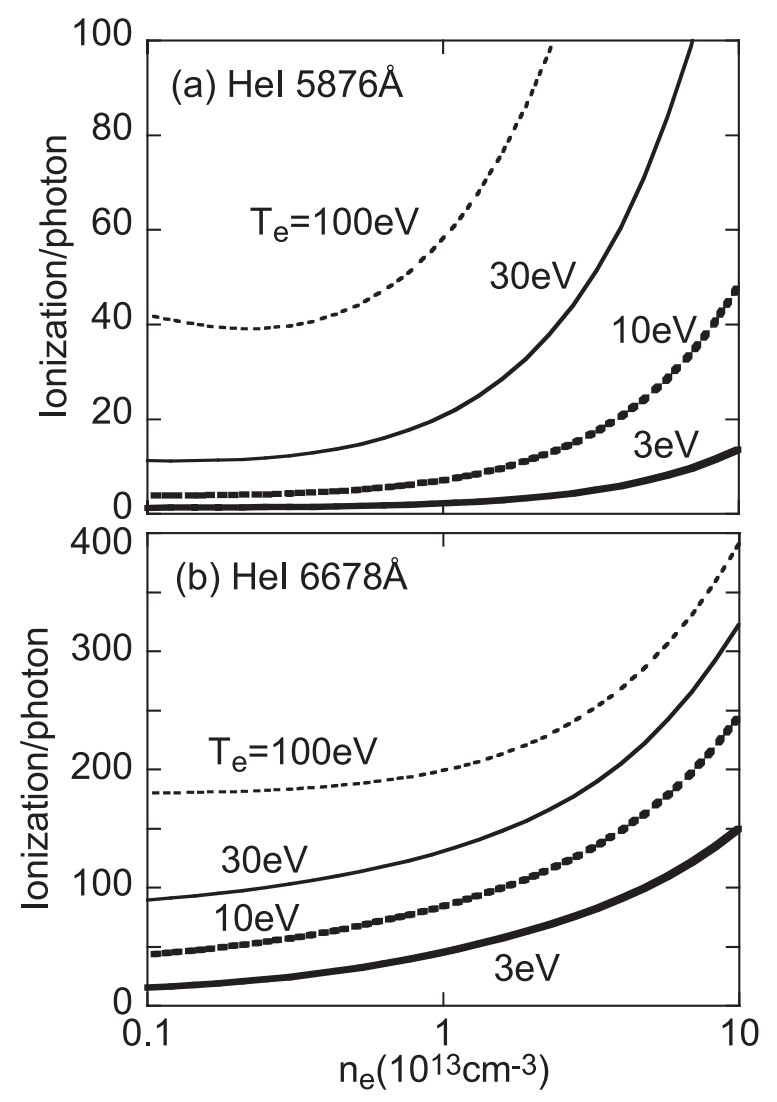

Fig. 2 Ionization events per photon for HeI emissions of (a) $2 \mathrm{p}^{3} \mathrm{P}-3 \mathrm{~d}^{3} \mathrm{D}: 5876 \AA$ and (b) $2 \mathrm{p}^{1} \mathrm{P}-3 \mathrm{~d}^{1} \mathrm{D}: 6678 \AA$ as a function of electron density. Calculation is performed for four different electron temperatures (thick solid line: $3 \mathrm{eV}$, thick dashed line: $10 \mathrm{eV}$, thin solid line: $30 \mathrm{eV}$, and thin dashed line: $100 \mathrm{eV}$ ). 
to HeI emissions is taken into account. In particular, the tendency of the density effect correction is similar to the HeI $6678 \AA$ case. The low $T_{\mathrm{e}}$ of $3 \mathrm{eV}$, as seen in the figure, is not realistic in normal LHD discharges except for the recombining phase. The temperature dependence is still seen in the ratio. In most discharges, however, the local electron temperature of the emissions distributes around $30 \mathrm{eV}$. Although the temperature dependence gives 20-30\% uncertainty for the ratio, the analysis can be performed practically without consideration of the edge parameter variations.

The triplet transition (HeI $5876 \AA$ ) of neutral helium is really strong compared to other singlet transitions like HeI $6678 \AA$. The intensity of HeI $5876 \AA$ is always 5-10 times stronger than other HeI lines in LHD. The ratio of hydrogen ion flux to total ion flux for HeI $5876 \AA$, on the other hand, has a relatively strong density and temperature dependences compared to HeI $6678 \AA$. Taking into account only the ratio itself, the use of HeI $6678 \AA$ is of course better than $5876 \AA$. In the wavelength range near $6678 \AA$, however, other relatively strong emission lines exist in addition to the $\mathrm{H}_{\alpha}$ emission. Therefore, HeI $5878 \AA$ is used in
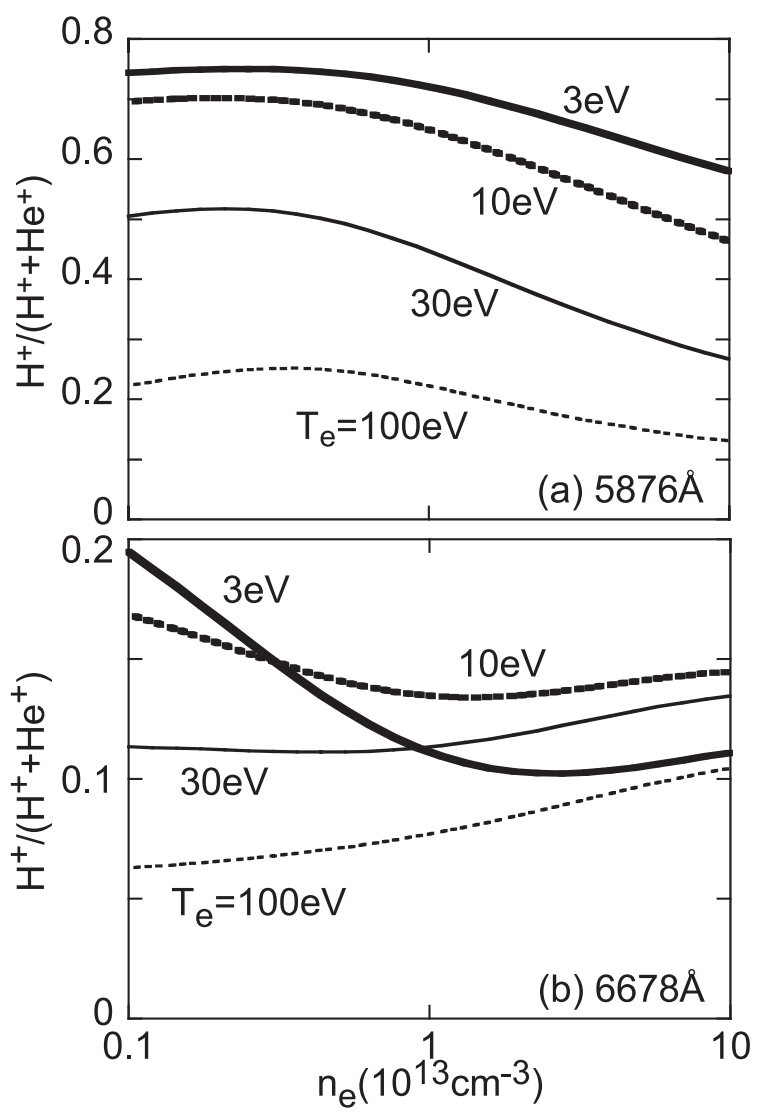

Fig. $3 \mathrm{H}^{+} / \mathrm{H}^{+}+\mathrm{He}^{+}$ratio (三 ratio of hydrogen ion flux to total ion flux $)$ as a function of electron density at $\varepsilon_{\mathrm{H}_{\alpha}}=\varepsilon_{\mathrm{HeI}}(\varepsilon$ : emissivity); (a) HeI $5876 \AA$ and (b) HeI $6678 \AA$ A. Calculation is performed for four different electron temperatures (thick solid line: $3 \mathrm{eV}$, thick dashed line: $10 \mathrm{eV}$, thin solid line: $30 \mathrm{eV}$, and thin dashed line: $100 \mathrm{eV}$ ). the present measurement in order to increase the $\mathrm{S} / \mathrm{N}$ ratio of the signal.

\section{Measurement of $\mathrm{H}_{\alpha} / \mathrm{HeI}$ Ratio}

The view chords of the toroidal array in LHD are shown in Fig. 4 by thick dashed-dotted lines. A total of ten chords are installed on LHD, based on the toroidal pitch number of LHD. Three optical fibers with a core diameter of $0.3 \mathrm{~mm}$ are set with $f=10 \mathrm{~mm}$ lenses on an ICF152 vacuum flange quartz window. The view area of the optical fiber is approximately $15 \mathrm{~cm}$ in diameter at the plasma center. The visible light from LHD plasmas is transmitted to $\mathrm{H}_{\alpha}$ and $\mathrm{HeI}$ monitor assemblies through a 150 -m-long optical fiber. A schematic drawing of one of the monitor assemblies is shown in Fig. 5. The assembly consists of a total of 26 FC-type fiber connectors (13 each for signal input and output) with $f=10 \mathrm{~mm}$ lenses and an interference filter with a diameter of $100 \mathrm{~mm}$. In Fig. 5, half of the fiber connectors for converting into parallel light are seen on the signal input side. The $\mathrm{H}_{\alpha}$ or HeI emission selected by the interference filter is collected at the signal output side using the same fiber connectors and is detected by photomultipliers through short optical fibers $(3 \mathrm{~m})$ with the same diameter as the input side. The interference filter can be easily replaced by another filter from the top of the assembly.

Since a large interference filter is used, the transmission rate of the line is a little different for each fiber due to the spatially nonuniform transmission rate. Typical transmission rates of the filters for $\mathrm{H}_{\alpha}$ and HeI are shown in Figs. 6 (a) and (b), respectively. The full width at half max-

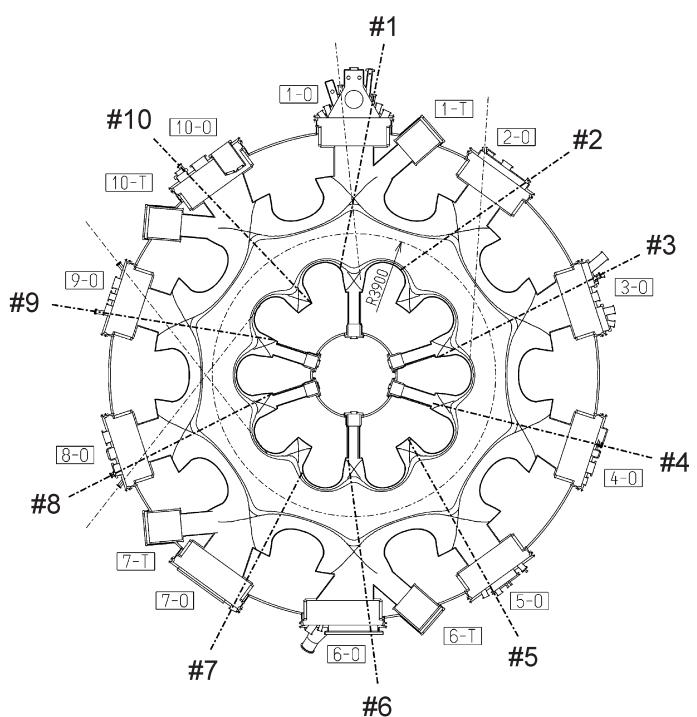

Fig. 4 Toroidal array in LHD for $\mathrm{H}_{\alpha}(6563 \AA)$ and HeI (5876 ̊) monitors. Ten observation chords are indicated with thick dashed-dotted lines. Numbers indicate each helical section (toroidal pitch number of LHD: $m=10$ ). Three tangential observation chords traced with thin dashed-dotted lines are installed as a monitor for heating devices. 


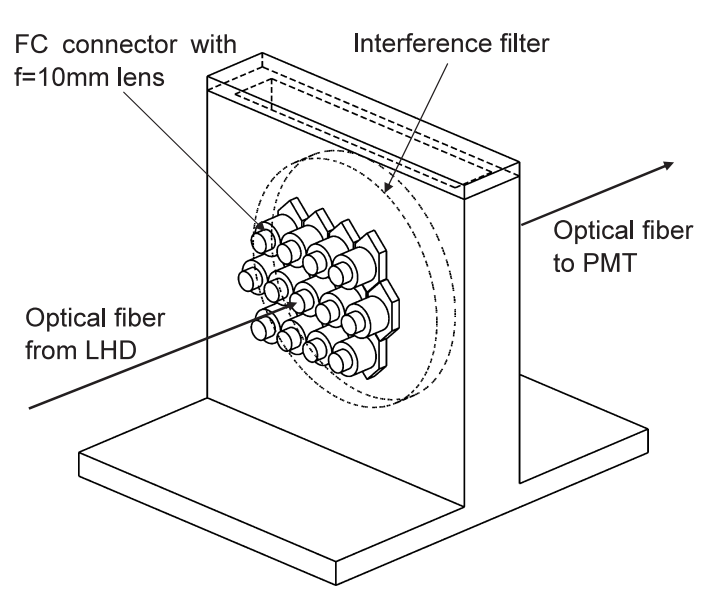

Fig. $5 \mathrm{H}_{\alpha}$ (or HeI) monitor assembly for toroidal array. Optical fibers (core diameter: $0.3 \mathrm{~mm}$ ) are set with $f=10 \mathrm{~mm}$ lens to produce parallel light. $\mathrm{H}_{\alpha}$ (or $\mathrm{HeI}$ ) signal selected by interference filter with diameter of $100 \mathrm{~mm}$ is collected by the same lens $(f=10 \mathrm{~mm})$ as input side and transferred to photomultipliers (PMTs) through optical fibers.
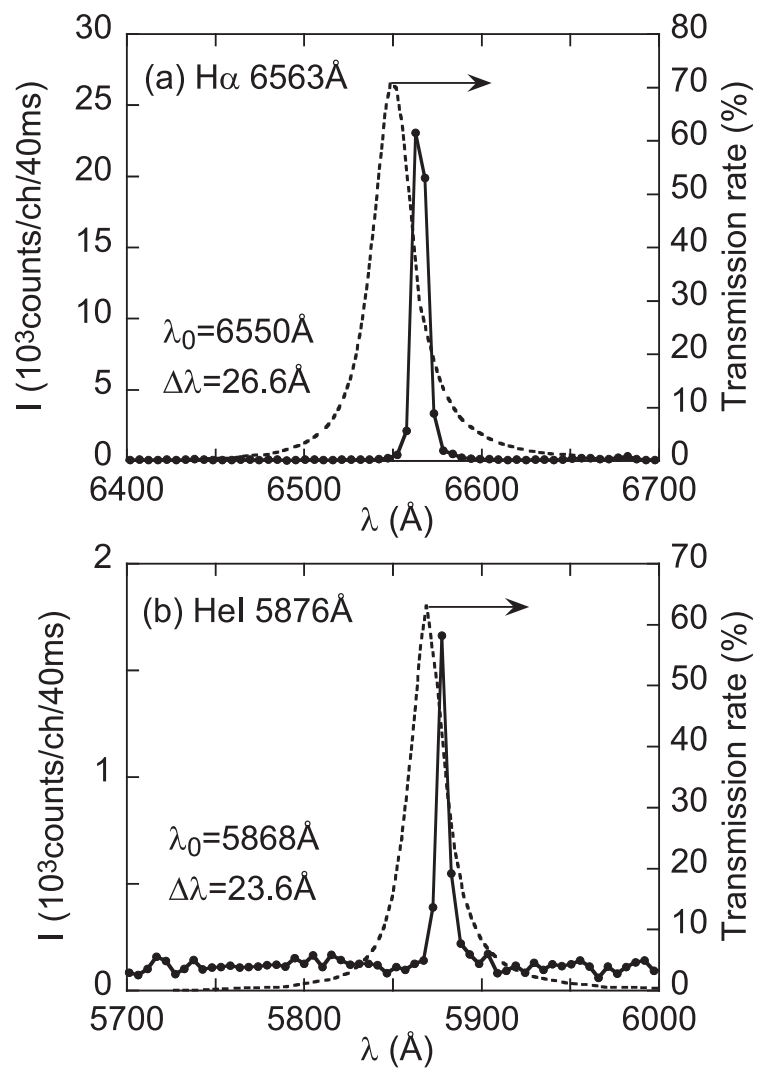

Fig. 6 Transmission rates (dashed lines) of interference filters and spectra (solid lines) for (a) $\mathrm{H}_{\alpha}$ and (b) HeI lines.

imum (FWHM) of the filter response is $\Delta \lambda=26.6 \AA$ at $\mathrm{H}_{\alpha}$ $6653 \AA$ and $\Delta \lambda=23.6 \AA$ at HeI $5876 \AA$. It is difficult to adjust all the signals to the central position of the filter response because of its nonuniformity. However, each channel keeps, at least, the transmission rate of 30-50\% against

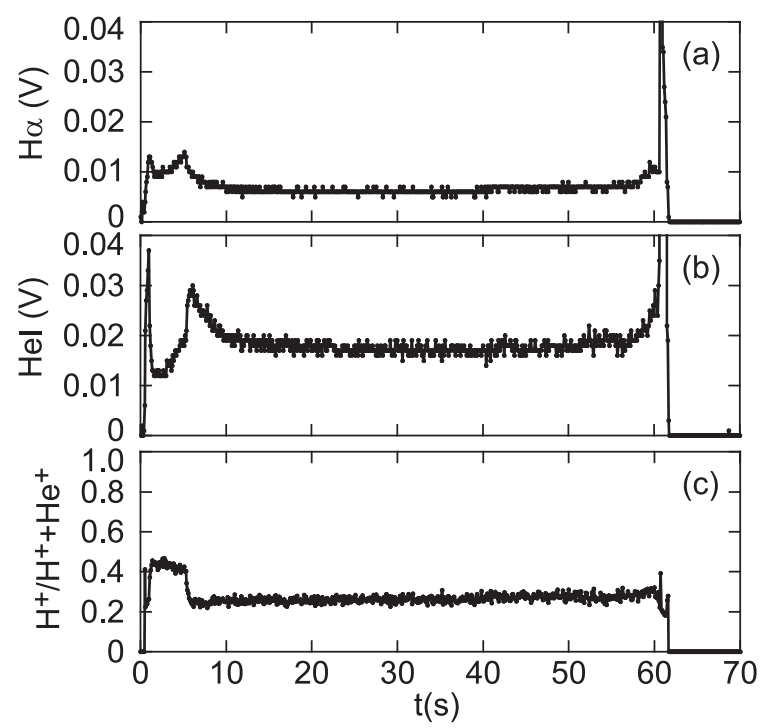

Fig. 7 Example of $\mathrm{H}^{+} / \mathrm{H}^{+}+\mathrm{He}^{+}$ratio measurement in ICRF long-pulse $\mathrm{H}$-minority heating discharge maintained for $60 \mathrm{~s}$, which evidently demonstrates a steady $\mathrm{H}^{+} / \mathrm{H}^{+}+\mathrm{He}^{+}$ ratio appropriate for ICRF heating: (a) $\mathrm{H}_{\alpha}$ signal, (b) $\mathrm{HeI}$ signal, and (c) $\mathrm{H}^{+} / \mathrm{H}^{+}+\mathrm{He}^{+}$ratio.

the primary line intensity from the optical fiber. It seems that the transmission rates have been stable during the past 9 years.

The $\mathrm{H}^{+} / \mathrm{H}^{+}+\mathrm{He}^{+}$ratio is thus obtained by integrating 10 signals from the toroidal array observing the $\mathrm{H}_{\alpha}$ and $\mathrm{HeI}$ emissions. A typical result of $\mathrm{H}^{+} / \mathrm{H}^{+}+\mathrm{He}^{+}$ratio measurement is shown in Fig. 7 in the ICRF long-pulse discharge with minority heating [11]. The ratio can be stably adjusted to $20 \%$, which is favorable for efficient minority ion heating through a selective heat deposition to proton.

On the other hand, the present system becomes useless for a few types of LHD discharges, such as extremely highdensity operation with $\mathrm{H}_{2}$ pellet injection and $\mathrm{Ne}$-seeded discharges. In case of $\mathrm{H}_{2}$ pellet injection, the increase in the background continuum mainly formed by enhanced bremsstrahlung emission is considerably large, since the electron density sufficiently exceeds $10^{14} \mathrm{~cm}^{-3}$ and is close to $10^{15} \mathrm{~cm}^{-3}$ at the plasma center, and the electron temperature considerably reduces, i.e., down to $0.3-1.0 \mathrm{keV}$. Then, the output signal from the HeI filter involves a large amount of the continuum signal whereas the HeI line intensity is not changed. A typical HeI spectrum with an enhanced continuum level is shown in Fig. 8.

The $\mathrm{H}^{+} / \mathrm{H}^{+}+\mathrm{He}^{+}$ratio in discharges with $\mathrm{H}_{2}$ pellet injection was then examined in detail using a visible spectrometer with a CCD detector to check the interference filter system. The results are shown in Fig. 9. Nine $\mathrm{H}_{2}$ pellets are injected during $t=0.65-0.9 \mathrm{~s}$ in the discharge [12], and are indicated with arrows. In the spectrometer signals (solid lines), only the line intensity is calculated by subtracting the background continuum. There appears a large discrepancy between the filter (dashed line) and spectrom- 


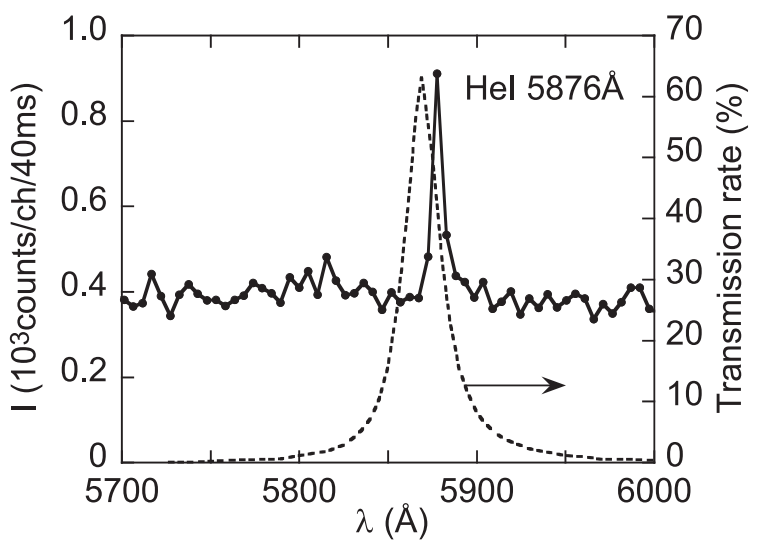

Fig. 8 HeI $5876 \AA$ A spectrum (solid line) during $\mathrm{H}_{2}$ pellet injection. Dashed line indicates filter response curve.

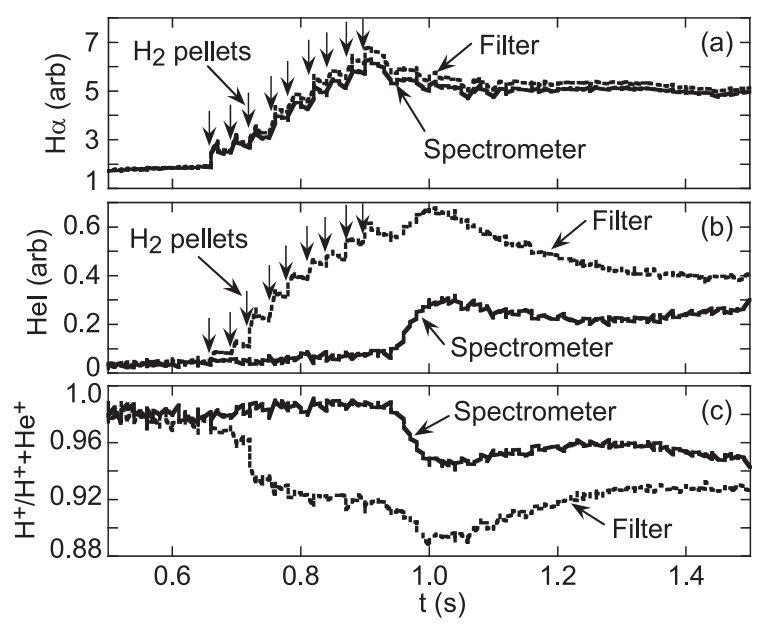

Fig. 9 (a) $\mathrm{H}_{\alpha}$ signal, (b) $\mathrm{HeI}$ signal, and (c) $\mathrm{H}^{+} / \mathrm{H}^{+}+\mathrm{He}^{+}$ratio measured with interference filter (dashed lines) and visible spectrometer (solid lines). Time at injection of nine repetitive $\mathrm{H}_{2}$ pellets is indicated with arrows.

eter intensities in the HeI signal (see Fig. 9(b)), whereas no sufficient difference is seen in the $\mathrm{H}_{\alpha}$ signals between the two (see Fig. 9 (a)). Therefore, it is understood that the $\mathrm{H}^{+} / \mathrm{H}^{+}+\mathrm{He}^{+}$ratio does not change during the pellet injection phase (see Fig.9(c)). After the injection, however, the increase of the $\mathrm{He}^{+}$flux is clearly seen at $t=0.95 \mathrm{~s}$, where the electron density begins to decrease and the ion flux on the divertor plates quickly begins to increase. The enhanced $\mathrm{He}^{+}$flux is released from divertor plates by collisions with the protons that have escaped from core plasma after a certain particle confinement time.

In case of Ne-seeded discharges, many lines appear near HeI $5876 \AA$, as shown in Fig. 10. The Ne-seeded discharges have been sometimes used in LHD for diagnostics and increases in temperatures and resultant increase in NBI-driven toroidal current, etc. It is impossible to measure the HeI line using only the interference filter method in such high- $Z$ discharges.

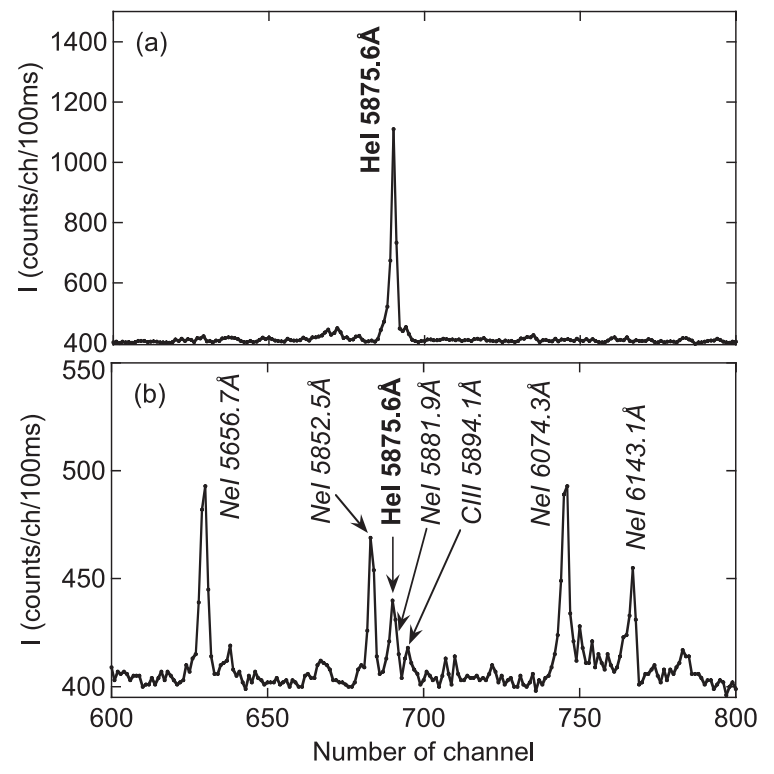

Fig. 10 Visible spectra near HeI $5876 \AA$ from He discharges (a) without and (b) with Ne puff.

\section{Experimental Results}

\section{1 $\mathrm{H}^{+} / \mathrm{H}^{+}+\mathrm{He}^{+}$ratio during past 9 years in LHD}

As an example of the measurement, the $\mathrm{H}^{+} / \mathrm{H}^{+}+\mathrm{He}^{+}$ ratio has been analyzed in almost all discharges from the 2nd cycle (1998) to the 10th cycle (2006). In these 9 years, the following different wall conditionings were attempted:

1st-\&2nd: daily He glow or ECR discharge (stainless steel divertor)

3rd: daily $\mathrm{H}_{2}$ or He glow discharge (carbon divertor) 4th-\&5th: daily He glow discharge (carbon divertor) 6th-\&7th: $\mathrm{H}_{2}(\mathrm{He})$ glow discharge before $\mathrm{H}_{2}(\mathrm{He})$ experiment

8th to -10th: He glow discharge only when necessary

Typical results are shown in Figs. 11 (a), (b), (c), and (d) for the $2 \mathrm{nd}, 3 \mathrm{rd}, 4 \mathrm{th}$, and 7 th cycles. The 1 st and 2 nd cycles (1998) had no carbon divertor plates and the discharges were operated with a stainless steel wall. NBI injection was started from the 2 nd cycle, although the 1 st cycle was operated by only ECH heating for roughly one month. In addition, the $\mathrm{H}_{2}$ and $\mathrm{He}$ discharges were repeated alternately in the 2 nd cycle. As a result, most of discharges were dominated by He ions. Before the 3rd cycle (1999), carbon divertor plates were newly installed on the vacuum wall $[1,13]$. Either $\mathrm{H}_{2}$ or $\mathrm{He}$ glow discharge was performed daily after the end of the day's experiment. In contrast, only He glow discharges were performed in the 4th cycle (2000). The difference is clearly visible in the two figures (see Figs. 11 (b) and (c)). After He glow discharges, any pure $\mathrm{H}_{2}$ discharges could not be maintained, although the $\mathrm{He}^{+}$flux gradually decreased according to shot repetition of $\mathrm{H}_{2}$ discharges. The additional $\mathrm{He}^{+}$flux is mainly 

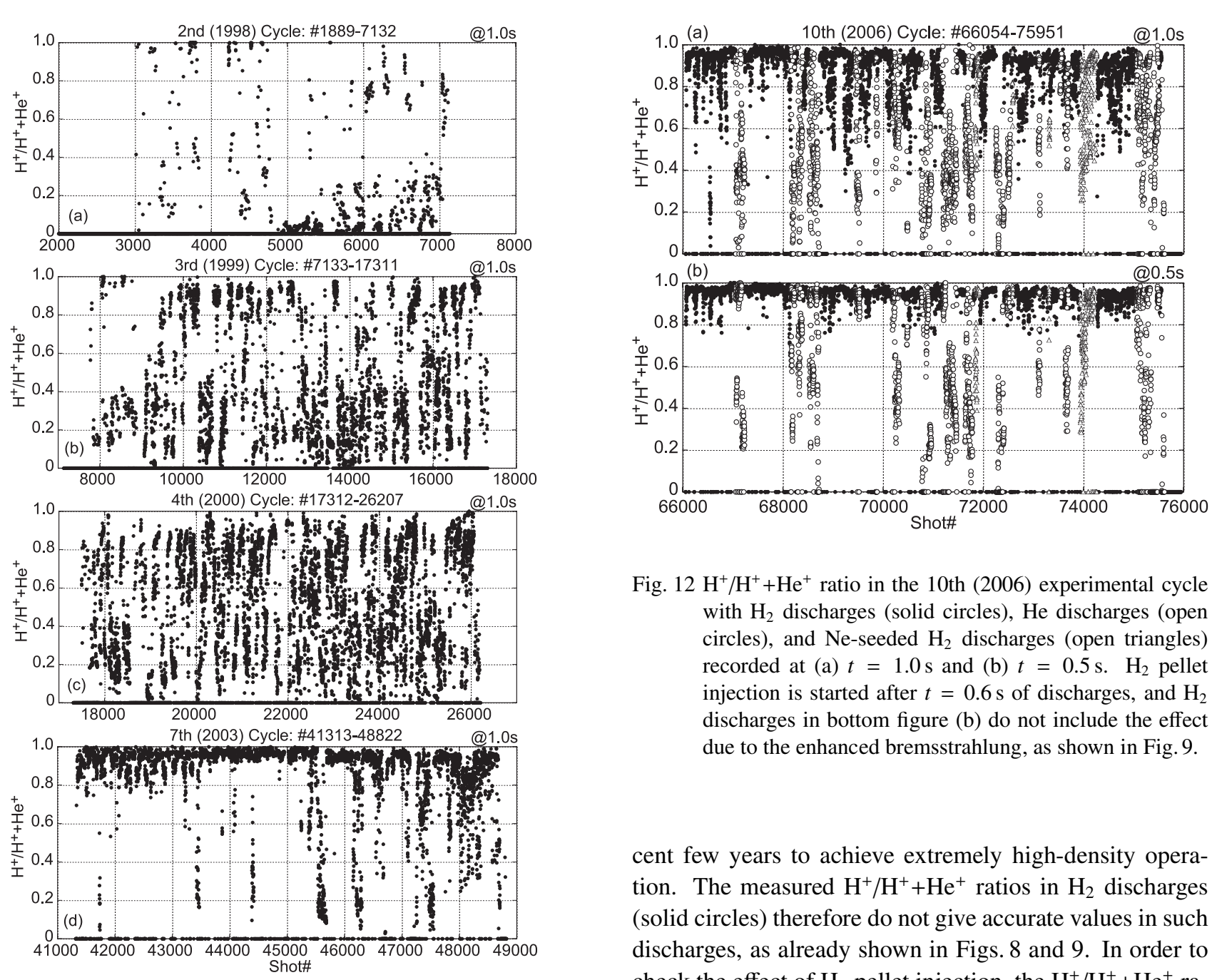

Fig. $12 \mathrm{H}^{+} / \mathrm{H}^{+}+\mathrm{He}^{+}$ratio in the 10th (2006) experimental cycle with $\mathrm{H}_{2}$ discharges (solid circles), He discharges (open circles), and $\mathrm{Ne}$-seeded $\mathrm{H}_{2}$ discharges (open triangles) recorded at (a) $t=1.0 \mathrm{~s}$ and (b) $t=0.5 \mathrm{~s} . \mathrm{H}_{2}$ pellet injection is started after $t=0.6 \mathrm{~s}$ of discharges, and $\mathrm{H}_{2}$ discharges in bottom figure (b) do not include the effect due to the enhanced bremsstrahlung, as shown in Fig. 9.

Fig. $11 \mathrm{H}^{+} / \mathrm{H}^{+}+\mathrm{He}^{+}$ratios in the (a) 2nd (1998), (b) 3rd (1999), (c) 4th (2000), and (d) 7th (2003) experimental cycles.

released from the carbon divertor plates. In the 7th cycle (2003), the $\mathrm{H}^{+} / \mathrm{H}^{+}+\mathrm{He}^{+}$ratio was drastically changed, as seen in Fig. 11 (d). The working gas used in the glow discharge was selected according to the fueling gas in the next day's experiment. Since the He experiment was not performed so frequently in the 7th cycle, almost pure $\mathrm{H}_{2}$ operation became possible in the $\mathrm{H}_{2}$ gas fueled discharges.

Recent LHD operation (8th to -10th cycles) can excellently maintain good $\mathrm{H}_{2}$ discharges with less $\mathrm{He}^{+}$flux. On the contrary, the maintenance of the pure He discharge becomes considerably difficult, because the number of NBI beam lines (at present NBI\#1 to NBI\#4) increased with further enhancement of hydrogen neutral flux. The hydrogen flux from NBI is generated by both beam proton and $\mathrm{H}_{2}$ gas inflow from the injector. Then, the increase in the $\mathrm{H}^{+} / \mathrm{H}^{+}+\mathrm{He}^{+}$ratio is frequently seen even in the He-fueled discharges.

The results from the 10th cycle (2006) are shown in Fig. 12 (a). NBI discharges with repetitive $\mathrm{H}_{2}$ pellet injection have been performed very frequently during the re-

cent few years to achieve extremely high-density operation. The measured $\mathrm{H}^{+} / \mathrm{H}^{+}+\mathrm{He}^{+}$ratios in $\mathrm{H}_{2}$ discharges (solid circles) therefore do not give accurate values in such discharges, as already shown in Figs. 8 and 9. In order to check the effect of $\mathrm{H}_{2}$ pellet injection, the $\mathrm{H}^{+} / \mathrm{H}^{+}+\mathrm{He}^{+}$ratios are analyzed at $t=0.5 \mathrm{~s}$ before the pellet injection, as shown in Fig. 12 (b). Since the NBI is usually injected at $t=0.3 \mathrm{~s}$ for the plasma startup, the time of $t=0.5 \mathrm{~s}$ just corresponds to the initial phase of discharges with density rising phase. It is clearly seen from Fig. 12 (b) that the $\mathrm{H}_{2}$ discharges are purely fueled by $\mathrm{H}^{+}$ions. The discharges plotted in Fig. 7 do not include the repetitive $\mathrm{H}_{2}$ pellet injection. The He discharges indicated with open circles mainly originated from ICRF minority heating experiments (H: minority, He: majority). The results from $\mathrm{Ne}$-seeded discharges (open triangles) also include a large uncertainty and of course are not correct. However, the estimation of the uncertainty is very difficult at present, because the contribution of the line emissions from neon is different according to each shot.

\subsection{Replacement of atoms on divertor plates}

The replacement between argon and hydrogen at the divertor plates has been measured in LHD. Figures 13 (a) and (b) show shot-by-shot temporal behaviors of ArXVII (3.9482 $\AA$ ) intensity from the plasma core and the $\mathrm{H}^{+} / \mathrm{H}^{+}+\mathrm{He}^{+}$ratio after replacing Ar discharges by $\mathrm{H}_{2}$ discharges, respectively. The Ar discharges were continued for roughly 100 shots at densities of $0.5 \leq n_{\mathrm{e}} \leq$ $1.5 \times 10^{13} \mathrm{~cm}^{-3}$ before $\mathrm{H}_{2}$ discharges were started, and 


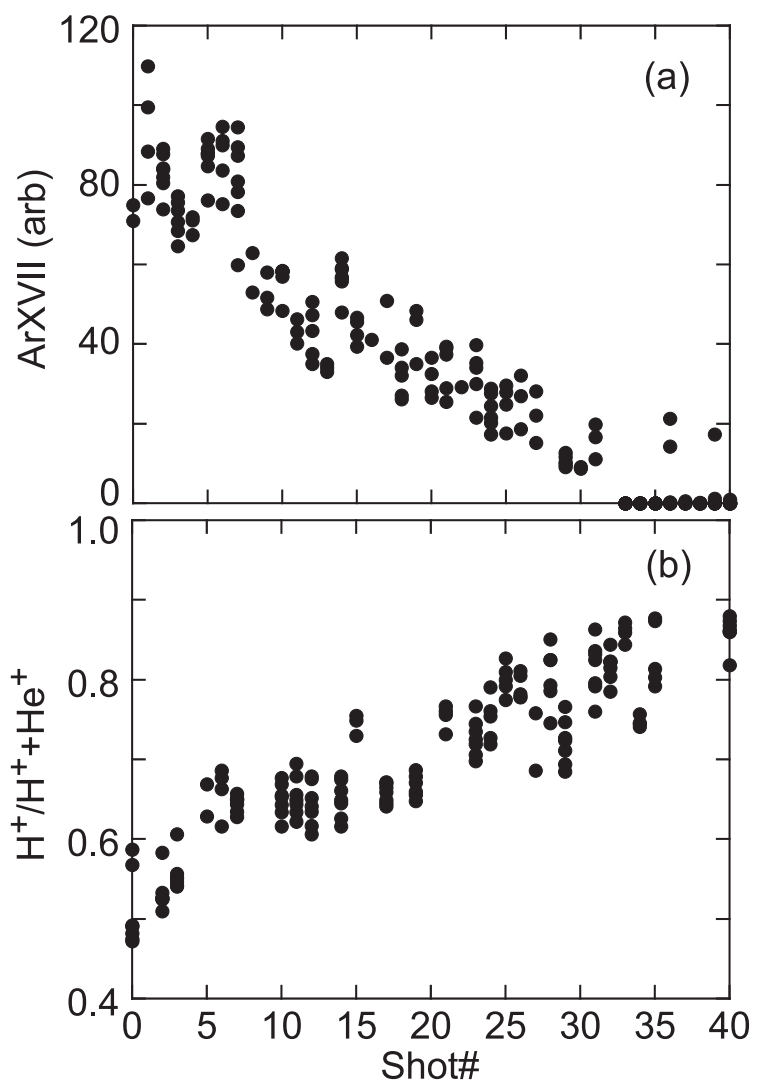

Fig. 13 Shot-by-shot changes in (a) intensity of ArXVII: $2.61 \AA$ and (b) $\mathrm{H}^{+} / \mathrm{H}^{+}+\mathrm{He}^{+}$ratio after replacing Ar NBI discharges with $\mathrm{H}_{2} \mathrm{NBI}$ discharges.

later the $\mathrm{H}_{2}$ discharges were operated at densities of $1.0 \leq$ $n_{\mathrm{e}} \leq 3.0 \times 10^{13} \mathrm{~cm}^{-3}$. The ArXVII intensity plotted in Fig. 13 (a) monotonically reduces after stopping the Ar discharge and completely disappears after 33 shots. On the contrary the $\mathrm{H}^{+} / \mathrm{H}^{+}+\mathrm{He}^{+}$ratio increases and reaches the saturation level of $\sim 0.83$ after 40 shots. This means that the Ar atoms deposited on the carbon divertor plates can be entirely replaced by hydrogen after accumulation of 30-40 shots of $\mathrm{H}_{2}$ discharges.

Finally, the hydrogen influx generated from the NBI devices has been measured $[14,15]$. The $\mathrm{H}^{+} / \mathrm{H}^{+}+\mathrm{He}^{+}$ratio measured from He NBI discharges operated at a constant density of $1 \times 10^{13} \mathrm{~cm}^{-3}$ is shown in Fig. 14 (a). The ratio is fixed to approximately 0.8 whereas only He puff is performed. This suggests the presence of additional hydrogen influx to the main plasma. On the other hand, we already presented in Fig. 7 that the ratio can be easily kept at an optimum low value of 0.2 in ICRF discharge. Then, the difference in the ratios between the NBI and ICRF He discharges was checked using the present method. For this purpose, the ICRF discharge was replaced by the He NBI discharge. The results are shown in Fig. 14 (b). The $\mathrm{H}^{+} / \mathrm{H}^{+}+\mathrm{He}^{+}$ratio just after the ICRF discharge was replaced by the He NBI discharge is plotted as a function of repeated shot numbers. Both the discharges were op-
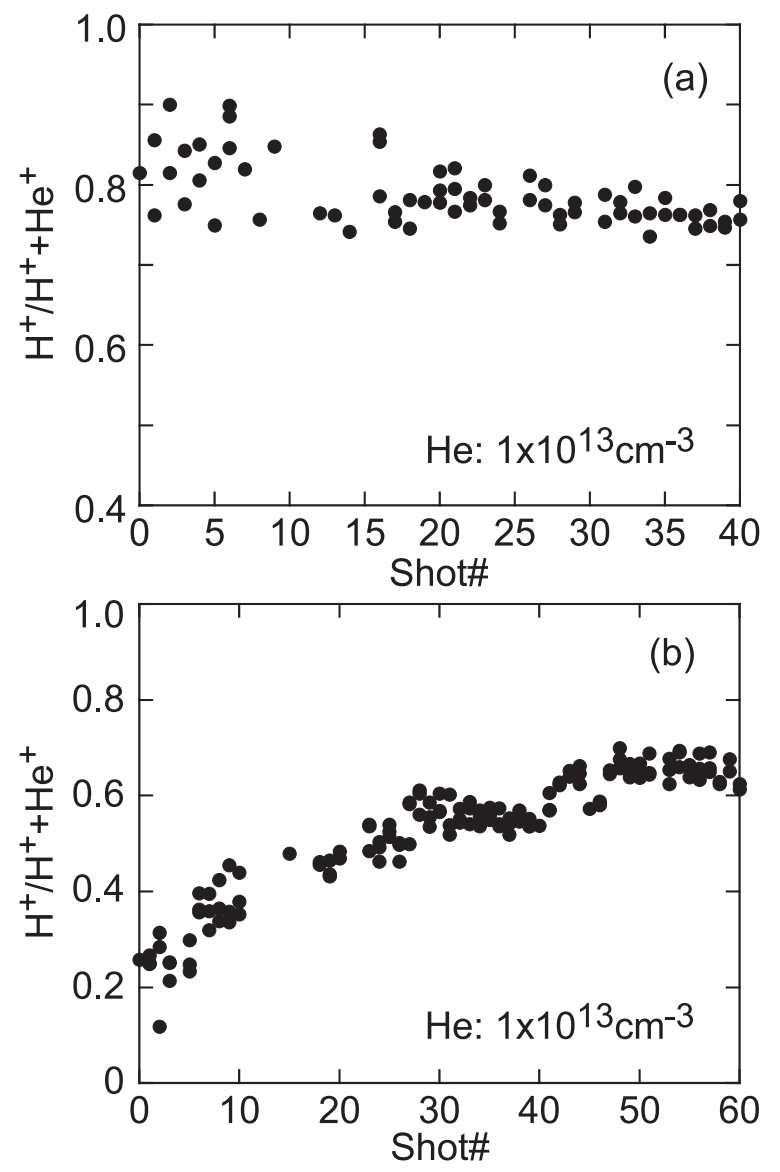

Fig. 14 Shot-by-shot changes in $\mathrm{H}^{+} / \mathrm{H}^{+}+\mathrm{He}^{+}$ratios (a) during $\mathrm{He}$ NBI discharges and (b) after replacing ICRF long-pulse $\mathrm{H}$-minority heating (working gas mixture: $\mathrm{H} / \mathrm{He}=20 \%$ ) discharges by He NBI discharges. Electron densities of He discharges are fixed at $1 \times 10^{13} \mathrm{~cm}^{-3}$ for both cases.

erated at a constant density of $1 \times 10^{13} \mathrm{~cm}^{-3}$. The ratio evidently increases from 0.2 to 0.65 whereas only He puff is performed. This increment of the ratio indicates hydrogen inflow from an NBI device, i.e., leaked $\mathrm{H}_{2}$ gas from NBI devices and injected proton beam. Further, it is seen that the He atoms deposited on the divertor plates can be replaced by the $\mathrm{H}$ atoms after accumulation of 40-50 shots of $\mathrm{H}_{2}$ discharges, which is quite similar to the Ar case.

\section{Summary}

A simple method for determining the ion flux ratio of hydrogen to helium was presented with measurement of intensity ratio of $\mathrm{H}_{\alpha}$ to HeI. The coefficients needed for the neutral influx ratio analysis are calculated using a collisional-radiative model. The analysis indicated that the influx ratio can be obtained directly from the intensity ratio of $\mathrm{H}_{\alpha}$ to $\mathrm{HeI}$ without consideration of edge density and temperature. The method was applied to the LHD discharges and the $\mathrm{H}^{+} / \mathrm{H}^{+}+\mathrm{He}^{+}$ratio was evaluated by integrating toroidally located $\mathrm{H}_{\alpha}$ and HeI signals. As a example of measurement, the $\mathrm{H}^{+} / \mathrm{H}^{+}+\mathrm{He}^{+}$ratios during the past 
9 years of LHD operation were represented with the history of glow discharges for wall conditioning and plasma facing components. The replacement of atoms deposited on the carbon divertor plates was also measured with the present method. The replacement can be completed by 4050 shot repetition of discharges. It is concluded that the present simple method on $\mathrm{H}^{+} / \mathrm{H}^{+}+\mathrm{He}^{+}$ratio measurement using the intensity ratio of $\mathrm{H}_{\alpha}$ to $\mathrm{HeI}$ can give useful information for machine operation.

\section{Acknowledgments}

The authors would like to thank all the members of the LHD experimental group. This study was performed under financial support for the LHD project (NIFS06ULPP527). This study was also partly supported by the JSPS-CAS Core-University Program in the field of "Plasma and Nuclear Fusion."

[1] S. Masuzaki, K. Akaishi, H. Funaba et al., J. Nucl. Mater. 290, 12 (2001).

[2] N. Ashikawa, S. Masuzaki, K. Nishimura et al., Fusion Eng. Design 81, 2831 (2006).
[3] N. Ashikawa, K. Kizu, J. Yagyu et al., J. Nucl. Mater. 363365, 1352 (2007).

[4] T. Morisaki, K. Narihara, S. Masuzaki et al., J. Nucl. Mater. 313-316, 548 (2003)

[5] S. Morita, T. Morisaki, M. Goto et al., Nucl. Fusion 47, 1033 (2007).

[6] M. Goto, K. Sawada and T. Fujimoto Phys. Plasmas 9, 4316 (2002).

[7] M. Goto, S. Morita, K. Sawada et al., Phys. Plasmas 10, 1402 (2003).

[8] M. Goto and S. Morita, Phys. Rev. E 65, 026401 (2002).

[9] K.J. Behringer, J. Nucl. Matter. 145-147, 145 (1987).

[10] S. Morita, H. Yamada, R. Akiyama et al., Fusion Technol. 27, 239 (1995).

[11] T. Mutoh, R. Kumazawa, T. Seki et al., Nucl. Fusion 47, 1250 (2007).

[12] R. Sakamoto, H. Yamada, Y. Takeiri et al., Nucl. Fusion 46, 884 (2006).

[13] S. Morita, M. Goto, H. Funaba et al., Physica Scripta T91, 48 (2001).

[14] Y. Takeiri, O. Kaneko, K. Tsumori et al., Rev. Sci. Instrum. 71, 1225 (2000).

[15] K. Tsumori, K. Nagaoka, M. Osakabe et al., Rev. Sci. Instrum. 75, 1847 (2004). 